as soon as they appear. They demand at once strict antiseptic treatment.

The same absolutely antiseptic condition of the fingers of the attendant is requisite when the nipples are handled, as when any operation in connection with genito-urinary apparatus of the puerperal woman is undertaken. Much the same responsibility attaches to the physician and the nurse with reference to mammary abscess, as in the case of puerperal fever. That is the physician and the nurse, provided the care of the patient has been completely in their hands, are responsible theoretically for the unfortunate accident. Either they have neglected to institute the precautions, plainly indi cated, or they have themselves infected nipples. This is not at all a pleasant doctrine for the practitioner, but is a very wholesome one for the patient.

When mastitis actually begins, the child must be removed from the affected breast immediately, and not at a time when the inflammation has already attained considerable proportions. "When this precaution is neglected," writes Olshausen, "or when it is not observed within the first twenty-four hours after the chill, which almost always characterizes the beginning of the disease, suppuration almost always follows. If the child is at once removed from the affected breast, in three-fourths, or more, of the cases, the mastitis will be cut short." In addition to the cessation of functional activity, the breast ought to be suspended, an ice-bag applied, and a purgative exhibited.

Olshausen has observed that when the fever continues longer than thirty-six hours, suppuration almost invariably results. This limit seems to us too brief, as certain cases of undoubted parenchymatous mastifls have come under our observation, in which resolutions has occurred even when the fever lasted as long as forty-eight or even seventy-two hours.

w. w. J.

\section{TWO NEW TEREBINTHINATES.}

The revival of interest in the terebinthinates of late has resulted in the extensive use of terebine in bronchial affections. Dr. Prosser James has recently called attention, in the Lancet, March Io, 1888 , to two other allied products, and more particularly to their use in diseases of the respiratory mucous tract. These are the essential oil of the mountain pine, and hydrate of terpin.

The Mountain Pine, Mugho, or Pinus pumilio of Lambert, is the tree from which exudes the Hungarian balsam, once so highly prized. By distilling the young branches with water a volatile oil is obtained, which has been known for a long time as "oleum templinum" or "Krummolzöl," and is the most potent agent in the so-called "pine-cure" of Reichenhall and other German spas. As the vapor of the water escapes into the inhalation rooms it is medicated with the volatile oil. By an improved process and apparatus, and care in selecting the material, a much finer product is now obtained-a very pure essential oil, oleum pini pumilionis, which has been imported into England under the name pumiline. It has the odor of the most fragrant variety of the pine, and is less irritating than other fir oils. When sprinkled or sprayed about a sick-room, says Dr. James, it imparts a lasting and grateful fragrance to the air, which is not oppressive, and seems to be disinfectant; or it may be thrown into a little warm water and allowed to evaporate; or it may be diffused by a steam inhaler, or a hand-ball atomizer.

This oil can be taken internally, and is admirable for inhalation. When prolonged inhalation is desired a few drops may be put in a suitable respirator, and patients frequently prefer it to other inhalants. For short steam inhalations from 10 to 20 drops may be put in to any common inhaler half full of water at $x 50^{\circ}$ to $x 60^{\circ} \mathrm{F}$, and the vapor inhaled; but it is better to diffuse the oil through water by magnesia, powdered silex, or prepared talc in the proportion of 40 to 60 minims of oil to 20 grains of magnesia and $r$ ounce of water. One teaspoonful of this mixture added to 8 ounces of cold water is stirred in the inhaler, and when 8 ounces of boiling water are added a convenient temperature is obtained; as it cools a little more boiling water is added, if it be desired to prolong the inhalation. This is milder than the B. P. vapor olei pini sylvestris, and may be used when that is too irritating. "It is a very mild stimulant to the mucous membrane, and an agreeable remedy for inhalation in relaxation, congestion and chronic catarrhal affections of the respiratory tract. Oleum pini pumilionis is also an excellent addition to other inhalations, such as creasote and various essential oils. It disguises the disagreeable odor of some and imparts its own fragrance to others." Dr. James has used it to disguise the smell of iodoform, but the mixture should be freshly prepared, as after a few days decomposition takes place with the liberation of free iodine. The oil mixes well with eucalyptol and other inhalants.

For internal use the dose of the oleum pini pumilionis is from $I$ to 5 minims, and small doses may be taken in lozenges or on a lump of sugar, just as terebine is often taken, or a tragacanth mixture may be 
made, or larger doses may be given in capsules. When taken internally the drug acts as a carminative. $\mathrm{It}$ is easily and quickly absorbed, and in the blood and tissues probably behaves like other terebinthinates. "It is its action on the bronchial membrane during excretion that renders it valuable in disease of this surface, being a stimulant, expectorant, and disinfectant, hence indicated in chronic bronchitis, dilatation of bronchi, bronchorrhœa, some states of phthisis, and other affections. The effect on the kidneys, however, should not be forgotten. The violet odor of the urine is produced as with some other terebinthinates, and small doses seem diuretic, but its use in diseases of the urinary passages manifestly requires care." It may be used externally sprinkled on flannel, or on spongio-piline. It is said to be a cleanly, prompt, and useful stimulant and counter-irritant, and sometimes appears to have slight anæsthetic properties.

The second terebinthinate, terpin hydrate, is very different from the oleum pini pumilionis. It has no odor, but little taste, is rather insoluble, and is solid. When crystallized spontaneously from a mixture of turpentine and water it appears as small needles, or it may be obtained in large rhombic crystals by allowing 3 parts of alcohol, 4 of turpentine, and $x$ of nitric acid to stand in shallow dishes for 3 or 4 days. It is only sparingly soluble in cold water or tupentine, but more soluble in hot-water, alcohol and ether; on account of its slight solubility it is best given in pill or wafer. It may be dissolved in warm glycerin, and then an equal quantity of some syrup may be added, which makes a suitable linctus. From 16 to 24 grains to $x$ ounce gives 2 to 3 grains to the teaspoonfull, a dose that can be taken every 3 or 4 hours. Small doses, such as the above, should be used at first, since they are often sufficient for the bronchial affection, and may affect the kidneys, for large doses given to animals have caused hæmaturia. The effects of terpin hydrate are similar to those of other terebinthinates, and it is useful sometimes as a substitute for them on account of being tasteless and odorless.

It restrains the cough and secretion of bronchitis, stimulating the bronchial membrane to healthier action; and Germain Sée has found full doses restrain the copious sputa of some cases of phthisis, and had no case of gastric disturbance after long use of the drug, though others have been less fortunate. In some cases small doses seem to increase the bronchial secretion. It has been used successfully in hæmoptysis, and in some cases it is mildly diuretic. Its alleged hypnotic property is probably due to the rapid relief to the cough.

W. G. E.
RELATION OF SCROFULOUS GLAND DISEASE TO OTHER FORMS OF TUBERCULOSIS.

Mr. Frederick S. Eve has recently published a series of experiments (in the British Medical Journal, of April r 4, r888) undertaken for the purpose of determining the relation of scrofulous gland disease to other forms of tuberculosis. It will be remembered that some experiments by M. S. Arloing appeared to prove that inoculation with material from scrofulous glands was innocuous to rabbits, while it produced general tuberculosis in guinea pigs. From his experiments he inferred that either scrofula and tuberculosis were nearly allied affections, but caused by different agents, or they were derived from a single virus, of which the activity was modified in the scrofulous form.

Mr. Eve's experiments were made with small fragments of the glands prepared with sterilized instruments, "and the possibility of infection of the animals with true tuberculosis was negatived by the precautions taken." In his experiments the inoculations uniformly caused tuberculosis. While he shows that the virus of strumous giand disease produces visceral tuberculosis in rabbits as well as in guineapigs, he admits that the disease in rabbits is not so acute and rapidly fatal as that following inoculation with acute miliary tubercle. "The difference is one of degree, not of a kind permitting us to infer, with Arloing, that struma is a specialized form of the tuberculous virus. We must therefore fall back on another explanation of the clinically innocent course of strumous gland disease; and we find it probably in the locality or soil in which the virus is implanted. Taking cervical gland disease, if the virus is not inherited from a consumptive or strumous parent, it may be surmised that the common bacillus of phthisis is implanted in the lymph follicles of the pharynx or tonsils, in one of the adjoining mucous membranes on the skin, and is carried direct to the glands. These, acting as filters, arrest its progress permanently, except in some cases in which suppuration and ulceration take place, when the surrounding textures may become implicated, and general dissemination ensue. The malignancy of the virus may be somewhat attenuated under the local influence of the lymph-cells and leucocytes in the gland, but to admit that the virus producing the disease is $a b$ initio specialized, would be to infer that the strumous disease could only be produced by the virus of struma, and no other." Mr. Eve emphasizes the objections to the terms "scrofula" and "struma" for lesions resembling tuberculosis. The evidence that tends to connect strumous gland disease more 